\title{
Review Article \\ Vacuum Condensates as a Mechanism of Spontaneous Supersymmetry Breaking
}

\author{
Antonio Capolupo and Marco Di Mauro \\ Dipartimento di Fisica E.R.Caianiello, Universitá di Salerno and INFN Gruppo Collegato di Salerno, 84084 Fisciano, Italy \\ Correspondence should be addressed to Antonio Capolupo; capolupo@sa.infn.it
}

Received 27 April 2015; Revised 23 June 2015; Accepted 1 July 2015

Academic Editor: Ignatios Antoniadis

Copyright (C) 2015 A. Capolupo and M. Di Mauro. This is an open access article distributed under the Creative Commons Attribution License, which permits unrestricted use, distribution, and reproduction in any medium, provided the original work is properly cited. The publication of this article was funded by SCOAP ${ }^{3}$.

\begin{abstract}
A possible mechanism for the spontaneous breaking of SUSY, based on the presence of vacuum condensates, is reviewed. Such a mechanism could occur in many physical examples, at both the fundamental and emergent levels, and would be formally analogous to spontaneous SUSY breaking at finite temperature in the TFD formalism, in which case it can be applied as well. A possible experimental setup for detecting such a breaking through measurement of the Anandan-Aharonov invariants associated with vacuum condensates in an optical lattice model is proposed.
\end{abstract}

\section{Introduction}

Supersymmetry (SUSY) $[1,2]$ has had a huge impact on contemporary physics, not only from the purely theoretical and mathematical points of view, but also from the phenomenological and experimental ones. This is despite the absence, up to now, of any clear experimental signature of its existence at the fundamental level. The main reason for this is that to date SUSY provides the best available explanation of the gauge hierarchy problem of the Standard Model [3], as well as providing candidates for dark matter and improving the situation of the dark energy issue (which however is still far from settled). In a few years, the situation may radically change due to the results coming from the LHC, but it is a fact that if SUSY exists at a fundamental level, it must be broken, either spontaneously or explicitly, since otherwise the superpartners of the known particles would be degenerate with the latter and thus would have been observed long ago. For this reason over the years there has been a lot of activity concerning SUSY breaking, and in particular the spontaneous breaking case (see, e.g., $[4,5]$ and references therein).

Besides fundamental SUSY, an interesting possibility, both on its own right and as a way to experimentally test ideas on SUSY and its breaking in the near future, is emergent SUSY.
Namely, it is possible that a condensed matter system may display SUSY at low energies, which may or may not be spontaneously broken. In particular, relativistic supersymmetric theories could be simulated with cold atom systems in optical lattices [6]. In what follows, we will describe a mechanism for SUSY breaking, based on vacuum condensates, which may be valid both at a fundamental level and at an emergent level $[7,8]$. The latter possibility also suggests ways to investigate this mechanism in table top experiments.

The idea is to exploit the formal analogy between thermal field theory in its Thermofield Dynamics (TFD) formulation [9] and different physical phenomena characterized by vacuum condensates similar to those appearing in the thermal context [10-15]. As in the thermal case, SUSY is spontaneously broken (see below); we expect that this happens in the same way also in these other phenomena. A possible experiment involving the measurement of the Anandan-Aharonov invariant associated with the vacuum condensate is also described.

Before explicitly stating our conjecture, let us briefly recall how SUSY is spontaneously broken in TFD.

It is well known that SUSY is spontaneously broken at any finite temperature $[16,17]$, with the fundamental reason being the different statistical behavior of bosons and fermions. Finite temperature physics can be formulated in 
a way which is equivalent to the standard ensemble based picture but which emphasizes the appearance of vacuum condensates. This formalism goes under the name of Thermofield Dynamics [9]. In this formalism vacuum condensates in the thermal ground state are conveniently described by means of Bogoliubov transformations, and thermal effects are encoded in the appearance of a new vacuum which is unitarily inequivalent to the zero temperature one. Thermal averages are then just vacuum expectation values with respect to this new vacuum. In the standard picture [16, 17], SUSY breaking is due to the fact that it is not possible to write down thermal averages in a way consistent with SUSY, while in the TFD picture it is due to the fact that the new vacuum acquires a nonvanishing energy density. This picture thus links thermal breaking of SUSY to the standard description of SUSY breaking, whose order parameter is precisely the vacuum energy density $[4,5]$. This last fact is as well known a straightforward consequence of the SUSY algebra: if the vacuum is not invariant under SUSY transformations, that is, $Q_{\alpha}|0\rangle \neq 0$, then (here $Q$ is the supercharge that generates SUSY transformations, $H$ is the Hamiltonian of the theory, and $C$ is the charge conjugation matrix)

$$
\langle 0|H| 0\rangle=\frac{1}{8}\left\langle 0\left|\operatorname{Tr}\left(C \gamma^{0}[Q, Q]_{+}\right)\right| 0\right\rangle \neq 0,
$$

while of course if the vacuum is invariant, then $\langle 0|H| 0\rangle=0$. Physically, this is due to the fact that the zero point energies of fermions and bosons cancel out; schematically,

$$
\begin{aligned}
H & =H_{\psi}+H_{B} \\
& \sim \sum_{\mathbf{k}, i}\left\{\omega_{\mathbf{k}, i}^{\psi}\left(N_{\mathbf{k}, i}^{\psi}-\frac{1}{2}\right)+\omega_{\mathbf{k}, i}^{B}\left(N_{\mathbf{k}, i}^{B}+\frac{1}{2}\right)\right\},
\end{aligned}
$$

and in a supersymmetric theory $\omega_{\mathbf{k}, i}^{\psi}=\omega_{\mathbf{k}, i}^{B} \equiv \omega_{\mathbf{k}, i}$. In the case of TFD, the condensates which are present in the thermal vacuum lift the vacuum energy. Such a lift is not canceled in a supersymmetric theory, thereby triggering the spontaneous breaking of SUSY.

The point is that the formalism of Bogoliubov transformations [18], on which this vacuum condensate based description of thermal physics is founded, is quite universal and describes vacuum condensates in a host of different quantum field theoretical (QFT) phenomena at various length scales, from fundamental to emergent models [19]. Examples of such phenomena include quantum fields in external fields, such as Schwinger [10] and Unruh [11] effects and examples from condensed matter physics such as the BCS theory of superconductivity [12] and graphene [13], mixing in particle physics $[14,15]$. (In the case of mixing the situation is slightly different, in that the Bogoliubov transformation is nested in a unitary transformation of the fields; however, this does not qualitatively change what we will say.) This leads to the conjecture that, in all these cases, when a supersymmetric extension is possible at the classical level, vacuum condensates lift the vacuum energy, thereby spontaneously breaking SUSY $[7,8]$. We give some evidence for this conjecture by considering the free Wess-Zumino model [20]. Despite its simplicity, this simple picture should give a good qualitative understanding of the vacuum of more complicated systems.

Considering the range of the phenomena described by this picture, this mechanism may occur at a fundamental level, for example, triggered by particle mixing, as proposed in $[21,22]$, or at an emergent level. The first possibility is very interesting from a phenomenological point of view and may be used for model building, while the latter possibility suggests, as said, the possibility of conceiving experimental measurements of the vacuum energy due to the condensates, therefore corroborating our conjecture [7]. This will be also the object of the present paper, in which the possibility of probing thermal spontaneous SUSY breaking through geometric invariants [23] will be explored. To be specific, the relevant quantity is the Anandan-Aharonov invariant [24], which has been shown to be a feature of phenomena characterized by vacuum condensates [25].

A few comments are in order. First of all, since vacuum condensates are a genuine field theoretical and nonperturbative phenomenon, this kind of SUSY breaking can occur only in QFT, and it is nonperturbative in nature (consistently with the fact that if SUSY is unbroken at tree level, it can only be broken at the nonperturbative level [4]). Second, while in what follows we will give evidence for our conjecture in a simple case, we do not address the issue of the dynamical origin of that breaking or, which is the same, of the origin of the vacuum condensates, which depends on the specific details of the phenomena under study and which in some cases such as mixing is to date unknown. The effective description of condensates in terms of Bogoliubov transformations is instead universal (besides being technically straightforward), since the form of this transformation is always the same, with the details of the specific case being encoded in the coefficients. This means that our discussion will be necessarily qualitative, while a more quantitative approach will need dealing with the complexities of the dynamics on various cases. In particular, the computation of quantities such as the scale of the breaking and mass differences between superpartners lies beyond the scope of the present paper. Also, we do not address the issue of the Goldstone fermion associated with the breaking. This issue, as well as the detailed study of some specific case, is left for some future publication.

\section{Vacuum Condensate and SUSY Breaking}

As a model of the supersymmetric extension of any of the above systems, we consider a situation in which SUSY is preserved at the Lagrangian level and study the vacuum condensation effects. These are described by a Bogoliubov transformation acting simultaneously, and with the same parameters, on the bosonic and on the fermionic degrees of freedom. This is required in order not to break SUSY explicitly. We conjecture that, in such a situation, SUSY is spontaneously broken by the appearance of vacuum condensates. In the present section, we collect some basic facts about Bogoliubov transformations in QFT (see, e.g., [19]), and then we prove in a simple case that vacuum condensates do shift the vacuum energy. 
The modes of any boson (fermion) field are described by a set of ladder operators $a_{\mathrm{k}}$, whose canonical (anti)commutation relations (CCRs) are $\left[a_{\mathbf{k}}, a_{\mathbf{p}}^{\dagger}\right]_{ \pm}=\delta^{3}(\mathbf{k}-\mathbf{p})$, with - for bosons and + for fermions and all other (anti)commutators vanishing. The vacuum $|0\rangle$ is defined by $a_{\mathbf{k}}|0\rangle$, and a Fock space is built out of it by acting with the creation operators $a_{\mathrm{k}}^{\dagger}$. form:

A generic Bogoliubov transformation has the following

$$
\tilde{a}_{\mathbf{k}}(\xi)=U_{\mathbf{k}} a_{\mathbf{k}}-V_{\mathbf{k}} a_{\mathbf{k}}^{\dagger}
$$

with the condition $\left|U_{\mathbf{k}}\right|^{2} \pm\left|V_{\mathbf{k}}\right|^{2}=1$, with - for bosons and + for fermions, ensuring the canonicity of the transformation. The transformation (3) is conveniently rewritten as $\widetilde{a}_{\mathbf{k}}(\xi)=$ $J^{-1}(\xi) a_{\mathbf{k}} J(\xi)$, where $J(\xi)$ is the generator which has the property $J^{-1}(\xi)=J(-\xi)$. The transformed operators $\widetilde{a}_{\mathbf{k}}(\xi)$ define a state $|\widetilde{0}(\xi)\rangle$ through $\widetilde{a}_{\mathbf{k}}(\xi)|\widetilde{0}(\xi)\rangle=0$, which is related to the vacuum $|0\rangle$ by $|\widetilde{0}(\xi)\rangle=J^{-1}(\xi)|0\rangle$. Such a state is a new vacuum of the system, for the following reason: the above transformation is a unitary operation if $\mathbf{k}$ assumes a discrete range of values, that is, if there is a finite or denumerably infinite number of CCRs. Then, the Fock spaces built on the two vacua $|0\rangle$ and $|\widetilde{0}(\xi)\rangle$ are equivalent. If on the other hand we assume that $\mathbf{k}$ has continuous infinity of values, which is the situation we are really interested in, we find that the transformation $|\widetilde{0}(\xi)\rangle=J^{-1}(\xi)|0\rangle$ is not unitary any more. This means that the two vacua and thus the two Fock spaces built over them are unitarily inequivalent. We thus have a family of states $|\widetilde{0}(\xi)\rangle$, each of which represents in principle a physical vacuum state for the theory. Of course, for these states to be true vacua of the system, the issue of stability should be addressed, but this depends on the specific system and is beyond the scope of this simple, free model.

Now, as announced, we will perform a Bogoliubov transformation on the free Wess-Zumino model and study its effects. The Lagrangian is given by (we adopt the notational conventions of [20])

$$
\begin{aligned}
\mathscr{L}= & \frac{i}{2} \bar{\psi} \gamma_{\mu} \partial^{\mu} \psi+\frac{1}{2} \partial_{\mu} S \partial^{\mu} S+\frac{1}{2} \partial_{\mu} P \partial^{\mu} P-\frac{m}{2} \bar{\psi} \psi \\
& -\frac{m^{2}}{2}\left(S^{2}+P^{2}\right),
\end{aligned}
$$

where $\psi$ is a Majorana spinor field, $S$ is a scalar field, and $P$ is a pseudoscalar field. This Lagrangian is invariant under the SUSY transformations:

$$
\begin{aligned}
& \delta S=i \bar{\kappa} \psi, \\
& \delta P=i \bar{\kappa} \gamma_{5} \psi, \\
& \delta \psi=\partial_{\mu}\left(S-\gamma_{5} P\right) \gamma^{\mu} \kappa-m\left(S+\gamma_{5} P\right) \kappa,
\end{aligned}
$$

where $\kappa$ is a Grassmann valued spinorial parameter.

We denote by $\alpha_{\mathrm{k}}^{r}, b_{\mathrm{k}}$, and $c_{\mathrm{k}}$ the annihilators for the fields $\psi, S$, and $P$, respectively, which annihilate the vacuum $|0\rangle=|0\rangle^{\psi} \otimes|0\rangle^{S} \otimes|0\rangle^{P}$ and we perform simultaneous
Bogoliubov transformations on the fermion and on the bosons:

$$
\begin{aligned}
& \widetilde{\alpha}_{\mathbf{k}}^{r}(\xi, t)=U_{\mathbf{k}}^{\psi}(\xi, t) \alpha_{\mathbf{k}}^{r}(t)+V_{-\mathbf{k}}^{\psi}(\xi, t) \alpha_{-\mathbf{k}}^{r \dagger}(t), \\
& \widetilde{b}_{\mathbf{k}}(\eta, t)=U_{\mathbf{k}}^{S}(\eta, t) b_{\mathbf{k}}(t)-V_{-\mathbf{k}}^{S}(\eta, t) b_{-\mathbf{k}}^{\dagger}(t), \\
& \widetilde{c}_{\mathbf{k}}(\eta, t)=U_{\mathbf{k}}^{P}(\eta, t) c_{\mathbf{k}}(t)-V_{-\mathbf{k}}^{P}(\eta, t) c_{-\mathbf{k}}^{\dagger}(t) .
\end{aligned}
$$

The Bogoliubov coefficients of scalar and pseudoscalar bosons are equal to each other: $U_{\mathbf{k}}^{S}=U_{\mathbf{k}}^{P}$ and $V_{\mathbf{k}}^{S}=V_{\mathbf{k}}^{P}$. We thus denote such quantities as $U_{\mathbf{k}}^{B}$ and $V_{\mathbf{k}}^{B}$, respectively. For fermions and for bosons, the Bogoliubov coefficients have the general form: $U_{\mathbf{k}}^{\psi}=e^{i \phi_{1 \mathbf{k}}} \cos \xi_{\mathbf{k}}(\zeta), V_{\mathbf{k}}^{\psi}=e^{i \phi_{2 \mathbf{k}}} \sin \xi_{\mathbf{k}}(\zeta)$, $U_{\mathbf{k}}^{B}=e^{i \gamma_{1 \mathbf{k}}} \cosh \eta_{\mathbf{k}}(\zeta)$, and $V_{\mathbf{k}}^{B}=e^{i \gamma_{2 \mathbf{k}}} \sinh \eta_{\mathbf{k}}(\zeta)$, respectively, where $\zeta$ represents the relevant parameter which controls the physics underlying the Bogoliubov transformation. For example, $\zeta$ is related to the temperature $T$ in Thermofield Dynamics and to the acceleration of the observer in Unruh effect case. Since the phases $\phi_{i \mathbf{k}}$ and $\gamma_{i \mathbf{k}}$, with $i=1,2$, are irrelevant, we neglect them.

The transformations (6) can be written at any time $t$ in terms of a generator $J(\xi, \eta, t)$; for example, for fermions we have

$$
\widetilde{\alpha}_{\mathbf{k}}^{r}(\xi, t)=J^{-1}(\xi, \eta, t) \alpha_{\mathbf{k}}^{r}(t) J(\xi, \eta, t),
$$

with similar relations holding for the bosonic annihilation and creation operators; in all of them, the generator is $J(\xi, \eta, t)=J_{\psi}(\xi, t) J_{S}(\eta, t) J_{P}(\eta, t)$, where $J_{\psi}, J_{S}$, and $J_{P}$ are the generator of the Bogoliubov transformations for fermion, scalar, and pseudoscalar fields [7].

The new vacuum is $|\widetilde{0}(t)\rangle=|\widetilde{0}(t)\rangle_{\psi} \otimes|\widetilde{0}(t)\rangle_{S} \otimes|\widetilde{0}(t)\rangle_{P}$, where the states $|\widetilde{0}(t)\rangle_{\alpha}$, with $\alpha=\psi, S, P$, are related to the original ones $|0\rangle_{\alpha}$ by the relations $|\widetilde{0}(t)\rangle_{\psi}=J_{\psi}^{-1}(\xi, t)|0\rangle_{\psi}$, $|\widetilde{0}(t)\rangle_{S}=J_{S}^{-1}(\eta, t)|0\rangle_{S}$, and $|\widetilde{0}(t)\rangle_{P}=J_{P}^{-1}(\eta, t)|0\rangle_{P}$, respectively; therefore, the full vacua are related by

$$
|\widetilde{0}(t)\rangle=J^{-1}(\xi, \eta, t)|0\rangle .
$$

We notice that $|\widetilde{0}(t)\rangle$ has the structure of a condensate of particles, and indeed we have

$$
\begin{aligned}
\left\langle\widetilde{0}(t)\left|\alpha_{\mathbf{k}}^{r \dagger} \alpha_{\mathbf{k}}^{r}\right| \widetilde{0}(t)\right\rangle & =\left|V_{\mathbf{k}}^{\psi}(\xi, t)\right|^{2} ; \\
\left\langle\widetilde{0}(t)\left|b_{\mathbf{k}}^{\dagger} b_{\mathbf{k}}\right| \widetilde{0}(t)\right\rangle & =\left\langle\widetilde{0}(t)\left|c_{\mathbf{k}}^{\dagger} \mathcal{c}_{\mathbf{k}}\right| \widetilde{0}(t)\right\rangle=\left|V_{\mathbf{k}}^{B}(\eta, t)\right|^{2} .
\end{aligned}
$$

Such a structure leads to an energy density different from zero for $|\widetilde{0}(t)\rangle$. To see this explicitly, we must compute the expectation value of the Hamiltonian $H$ corresponding to the Lagrangian in (4), which has the form $H=H_{\psi}+H_{B}$ (where $\left.H_{B}=H_{S}+H_{P}\right)$, on $|\widetilde{0}(t)\rangle$. The results for the two pieces of $H$ are given by

$$
\begin{aligned}
& \left\langle\widetilde{0}(t)\left|H_{\psi}\right| \widetilde{0}(t)\right\rangle=-\int d^{3} \mathbf{k} \omega_{\mathbf{k}}\left(1-2\left|V_{\mathbf{k}}^{\psi}(\xi, t)\right|^{2}\right), \\
& \left\langle\widetilde{0}(t)\left|H_{B}\right| \widetilde{0}(t)\right\rangle=\int d^{3} \mathbf{k} \omega_{\mathbf{k}}\left(1+2\left|V_{\mathbf{k}}^{B}(\eta, t)\right|^{2}\right),
\end{aligned}
$$


respectively. We thus obtain the final result

$$
\begin{aligned}
& \langle\widetilde{0}(t)|H| \widetilde{0}(t)\rangle \\
& \quad=2 \int d^{3} \mathbf{k} \omega_{\mathbf{k}}\left(\left|V_{\mathbf{k}}^{\psi}(\xi, t)\right|^{2}+\left|V_{\mathbf{k}}^{B}(\eta, t)\right|^{2}\right)
\end{aligned}
$$

which is different from zero and positive unless we are in the trivial case $\left|V_{\mathbf{k}}^{\psi}\right|^{2}=\left|V_{\mathbf{k}}^{B}\right|^{2}=0$.

The above computation clearly shows that the nonzero vacuum condensate energy, and thus the breaking of SUSY, is due to the fact that both the fermion and boson contributions to the condensate lift the vacuum energy by a positive amount, in contrast with the zero point energies which cancel each other.

\section{SUSY Breaking and the Anandan-Aharonov Invariant}

It has been shown that the presence of the AnandanAharonov invariant (AAI) [24], describing the time-energy uncertainty, characterizes the time evolution of the systems in which the vacuum condensate is physically relevant [25]. Then, AAIs could be used as a tool to reveal the SUSY breakdown [23]. The AAI appears in the evolution of any quantum state $\left|\chi_{\mathbf{k}}(t)\right\rangle$ which is not stationary; that is, its energy uncertainty $\Delta E_{\mathbf{k}}^{2}(t)=\left\langle\chi_{\mathbf{k}}(t)\left|H^{2}\right| \chi_{\mathbf{k}}(t)\right\rangle-\left(\left\langle\chi_{\mathbf{k}}(t)|H| \chi_{\mathbf{k}}(t)\right\rangle\right)^{2}$ must be nonzero. This is the case in the above listed instances [11-15]. When this condition is met, the AAI is defined as (we temporarily restore $\hbar) S_{\mathbf{k}}=(2 / \hbar) \int_{0}^{t} \Delta E_{\mathbf{k}}\left(t^{\prime}\right) d t^{\prime}$.

This invariant is analogous to the geometric phase (but it is defined for noncyclic and nonadiabatic evolution) and represents a time-energy uncertainty principle. It can be measured by studying interference of particles or by the analysis of the uncertainty on the outcome of measurements.

We consider the single particle states:

$$
\begin{aligned}
\left|\widetilde{\psi}_{\mathbf{k}}(\xi, t)\right\rangle & =\widetilde{\alpha}_{\mathbf{k}}^{r \dagger}(\xi, t)|\widetilde{0}(\xi, t)\rangle_{\psi}=J_{\psi}^{-1}(\xi, t)\left|\psi_{\mathbf{k}}\right\rangle, \\
\left|\widetilde{S}_{\mathbf{k}}(\eta, t)\right\rangle & =\widetilde{b}_{\mathbf{k}}^{\dagger}(\xi, t)|\widetilde{0}(\xi, t)\rangle_{S}=J_{S}^{-1}(\eta, t)\left|S_{\mathbf{k}}\right\rangle, \\
\left|\widetilde{P}_{\mathbf{k}}(\eta, t)\right\rangle & =\widetilde{c}_{\mathbf{k}}^{\dagger}(\eta, t)|\widetilde{0}(\eta, t)\rangle_{P}=J_{P}^{-1}(\eta, t)\left|P_{\mathbf{k}}\right\rangle .
\end{aligned}
$$

The energy variances of these states are $\Delta E_{\mathbf{k}}^{B}(t)=$ $\sqrt{2} \omega_{\mathbf{k}}\left|U_{\mathbf{k}}^{B}(\eta, t)\right|\left|V_{\mathbf{k}}^{B}(\eta, t)\right|$ and $\Delta E_{\mathbf{k}}^{\psi}(t)=\omega_{\mathbf{k}}\left|U_{\mathbf{k}}^{\psi}(\eta, t)\right|\left|V_{\mathbf{k}}^{\psi}(\eta, t)\right|$, respectively. Then, the corresponding AAIs are given by

$$
S_{\mathbf{k}}^{S}(t)=S_{\mathbf{k}}^{P}(t)=2 \sqrt{2} \int_{0}^{t} \omega_{\mathbf{k}}\left|U_{\mathbf{k}}^{B}\left(\eta, t^{\prime}\right)\right|\left|V_{\mathbf{k}}^{B}\left(\eta, t^{\prime}\right)\right| d t^{\prime}
$$

for scalar and pseudoscalar bosons and

$$
S_{\mathbf{k}}^{\psi}(t)=2 \int_{0}^{t} \omega_{\mathbf{k}}\left|U_{\mathbf{k}}^{\psi}\left(\xi, t^{\prime}\right)\right|\left|V_{\mathbf{k}}^{\psi}\left(\xi, t^{\prime}\right)\right| d t^{\prime},
$$

for the Majorana fermion field. Such invariants signal the presence of the condensate, since their values are controlled by the Bogoliubov coefficients and they vanish as the condensates disappear, that is, when $U_{\mathbf{k}}$ and $V_{\mathbf{k}}$ are zero.
(We notice that the particle mixing phenomenon is peculiar for the following reason. Although also in this case SUSY is spontaneously broken by condensate $[21,22]$, in this case the AAI arises mainly as an effect of the mixing of fields with only a small contribution due to the condensate structure $[26,27]$. Therefore, in this case the presence of the AAI is not directly linked with the presence of the condensate. In all the other cases instead the AAI is entirely due to the condensate contribution.)

Now we study the specific case of thermal states and propose a possible experiment to detect thermal SUSY violation by measuring nonvanishing AAIs. As is clear from all we said, in the TFD formalism [9], the thermal vacuum is a condensate generated through Bogoliubov transformations whose parameter is related to temperature. The Bogoliubov coefficients $U$ and $V$ have the general form [9] $U_{\mathbf{k}}=$ $\sqrt{e^{\beta \omega_{\mathbf{k}}} /\left(e^{\beta \omega_{\mathbf{k}}} \pm 1\right)}$ and $V_{\mathbf{k}}=\sqrt{1 /\left(e^{\beta \omega_{\mathbf{k}}} \pm 1\right)}$, with - for bosons and + for fermions, and $\beta=1 / k_{B} T$.

The energy variances of a temperature dependent single particle state are given by

$$
\Delta E_{\mathbf{k}}^{S}=\Delta E_{\mathbf{k}}^{P}=\sqrt{2} \omega_{\mathbf{k}} U_{\mathbf{k}}^{B} V_{\mathbf{k}}^{B}=\sqrt{2} \omega_{\mathbf{k}} \frac{e^{\beta \omega_{\mathbf{k}} / 2}}{\left(e^{\beta \hbar \omega_{\mathbf{k}}}-1\right)},
$$

for the bosonic states, and

$$
\Delta E_{\mathbf{k}}^{\psi}=\omega_{\mathbf{k}}^{\psi} U_{\mathbf{k}}^{\psi} V_{\mathbf{k}}^{\psi}=\hbar \omega_{\mathbf{k}}^{\psi} \frac{e^{\beta \omega_{\mathbf{k}}^{\psi} / 2}}{\left(e^{\beta \omega_{\mathbf{k}}^{\psi}}+1\right)},
$$

for the fermionic state. The corresponding AAIs are

$$
\begin{aligned}
& S_{\mathbf{k}}^{S}=S_{\mathbf{k}}^{P}=2 \sqrt{2} \omega_{\mathbf{k}} t \frac{e^{\beta \hbar \omega_{\mathbf{k}} / 2}}{e^{\beta \hbar \omega_{\mathbf{k}}}-1}, \\
& S_{\mathbf{k}}^{\psi}=2 \omega_{\mathbf{k}} t \frac{e^{\beta \hbar \omega_{\mathbf{k}} / 2}}{e^{\beta \hbar \omega_{\mathbf{k}}}+1} .
\end{aligned}
$$

In a supersymmetric model, at $T \neq 0$, the above invariants are different from zero.

\section{Experimental Realization}

The presence of the AAIs and then the SUSY violation could be tested by employing a mixture of cold fermion atoms and diatomic molecules trapped in two dimensional optical lattices [6], in which the Wess-Zumino model in $2+1$ dimensions can emerge at low energies. Such a system displays Dirac points in the Brillouin zone; therefore, the excitations will have relativistic dispersion relations and SUSY will be described by the super-Poincaré algebra, in contrast with other setups proposed in the literature, which display a nonrelativistic version of SUSY. The superpartner of the fermionic atom is a bosonic diatomic molecule. The setup allows simulating both the massless and the massive models, with the latter being attained by putting a Bose-Einstein condensate of dimolecules nearby, allowing exchange of pairs of molecules with the mixture through Josephson tunneling [6]. 


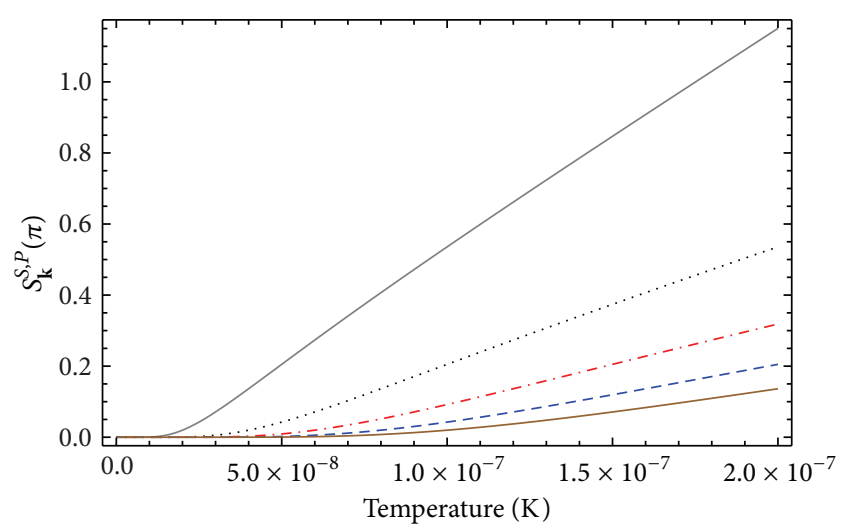

FIGURE 1: Plots of AAI for bosons, $S_{\mathbf{k}}^{S, P}$ as a function of temperature $T$, for a time interval $t=1 / \omega$ and for sample values of $\omega \in[2 \times$ $\left.10^{4} \mathrm{~s}^{-1}, 10^{5} \mathrm{~s}^{-1}\right], \omega=2 \times 10^{4} \mathrm{~s}^{-1}$ (gray solid line), $\omega=4 \times 10^{4} \mathrm{~s}^{-1}$ (black dotted line), $\omega=6 \times 10^{4} \mathrm{~s}^{-1}$ (red dot dashed line), $\omega=8 \times$ $10^{4} \mathrm{~s}^{-1}$ (blue dashed line), and $\omega=10^{5} \mathrm{~s}^{-1}$ (brown solid line).

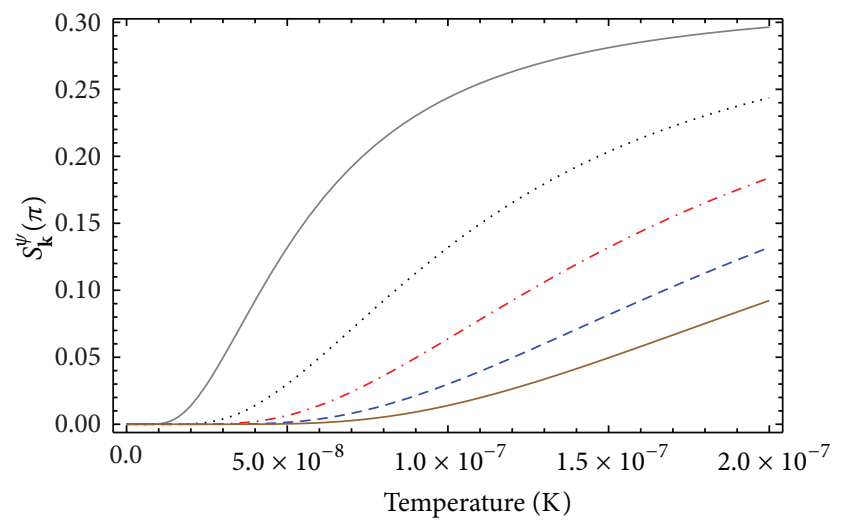

Figure 2: Plots of AAI for fermions, $S_{\mathbf{k}}^{\psi}$ as a function of temperature $T$, for the same time interval and sample values of $\omega$ as in Figure 1.

SUSY breaking is expected when the system is put at nonzero temperature. A proof of this breaking can come from the detection of a thermal Goldstone fermion, the phonino, which is predicted to appear in this case [2830]. This would present experimental difficulties; however, an alternative signal can come from the detection of the vacuum condensate. Building on [6], we propose measuring the difference between the geometric invariants in the evolution of two mixtures of cold atoms and molecules trapped in two coplanar, two-dimensional optical lattices, one at temperature $T \neq 0$ and the other one at $T=0$ [23]. Excitations of the lattice at $T \neq 0$ will be associated with the nonvanishing AAIs (18), while those in the $T=0$ lattice, on the other hand, have vanishing AAI. The presence of the condensate introduces a nontrivial modification on the uncertainty, therefore resulting in an inevitabe increase of the uncertainty on the outcome of measurements. Then, a study of the AAIs can be done also by analyzing the energy uncertainty in the lattice at $T \neq 0$.

Nontrivial values of the AAIs as functions of temperature are obtained (see Figures 1 and 2), by considering temperatures of the order of (20-200) nK, atomic excitation frequencies characteristic of Bose-Einstein condensates, that is, $\omega$ of order of $2 \times 10^{4} \mathrm{~s}^{-1}-10^{5} \mathrm{~s}^{-1}$ and time intervals of order of $t=1 / \omega[23]$. The values of the AAI we found are in principle detectable.

At temperatures above $\approx 200 \mathrm{nK}$, the condensate (and thus the AAIs) is expected to disappear. As a final comment, we notice that as happens for any system which presents a condensate structure [25], also in the present context, AAIs are unaffected by the presence of noise.

In conclusion, we have shown that, in the free WessZumino model, all the phenomena characterized by the presence of the vacuum condensate generate spontaneous SUSY breaking due to the nonzero vacuum energy. Indeed, bosons and fermion condensates both lift the vacuum energy by a positive amount. Such a breaking could be detected by measuring the AAIs generated by the condensates in a thermal bath in an optical lattice simulating the WessZumino model.

\section{Conflict of Interests}

The authors declare that there is no conflict of interests regarding the publication of this paper.

\section{Acknowledgments}

Partial financial support from MIUR and INFN is acknowledged.

\section{References}

[1] Y. A. Gol'fand and E. P. Likhtman, "Extension of the algebra of poincare group generators and violation of $\mathrm{P}$ invariance," JETP Letters, vol. 13, no. 8, p. 323, 1971, Pis'ma v Zh. Eksp. Teor. Fiz., vol. 13, p. 452, 1971.

[2] J. Wess and J. Bagger, Supersymmetry and Supergravity, Princeton Series in Physics, Princeton University Press, Princeton, NJ, USA, 2nd edition, 1992.

[3] S. Weinberg, "Ambiguous solutions of supersymmetric theories," Physics Letters B, vol. 62, no. 1, pp. 111-113, 1976.

[4] E. Witten, "Dynamical breaking of supersymmetry," Nuclear Physics B, vol. 188, no. 3, pp. 513-554, 1981.

[5] Y. Shadmi and Y. Shirman, "Dynamical supersymmetry breaking," Reviews of Modern Physics, vol. 72, no. 1, article 25, 2000.

[6] Y. Yu and K. Yang, "Simulating the Wess-Zumino supersymmetry model in optical lattices," Physical Review Letters, vol. 105, no. 15, Article ID 150605, 4 pages, 2010.

[7] A. Capolupo and M. Di Mauro, "Spontaneous supersymmetry breaking induced by vacuum condensates," Physics Letters A, vol. 376, no. 45, pp. 2830-2833, 2012.

[8] A. Capolupo and M. Di Mauro, "Vacuum condensates, flavor mixing and spontaneous supersymmetry breaking," Acta Physica Polonica B, vol. 44, no. 1, p. 81, 2013.

[9] Y. Takahashi and H. Umezawa, "Thermo field dynamics," Collective Phenomena, vol. 2, pp. 55-80, 1975, Reprinted in: International Journal of Modern Physics B, vol. 10, p. 1755-1805, 1996.

[10] J. Schwinger, "On gauge invariance and vacuum polarization," Physical Review, vol. 82, no. 5, pp. 664-679, 1951. 
[11] W. G. Unruh, "Notes on black-hole evaporation," Physical Review D, vol. 14, no. 4, pp. 870-892, 1976.

[12] J. Bardeen, L. N. Cooper, and J. R. Schrieffer, "Theory of superconductivity," Physical Review Letters, vol. 108, pp. 11751204, 1957.

[13] A. Iorio, "Weyl-gauge symmetry of graphene," Annals of Physics, vol. 326, no. 5, pp. 1334-1353, 2011.

[14] M. Blasone, A. Capolupo, O. Romei, and G. Vitiello, "Quantum field theory of boson mixing," Physical Review D, vol. 63, no. 12, Article ID 125015, 2001.

[15] M. Blasone, A. Capolupo, and G. Vitiello, "Quantum field theory of three flavor neutrino mixing and oscillations with CP violation," Physical Review D, vol. 66, no. 2, Article ID 025033, 2002.

[16] D. Buchholz and I. Ojima, "Spontaneous collapse of supersymmetry," Nuclear Physics B, vol. 498, no. 1-2, pp. 228-242, 1997.

[17] A. K. Das, Finite Temperature Field Theory, World Scientific, Singapore, 1997.

[18] N. N. Bogolyubov, "On the theory of superfluidity," Journal of Physics, vol. 11, no. 1, pp. 23-32, 1947, Izvestiya Akademii Nauk SSR, Seriya Fizicheskaya, vol. 11, pp. 77-90, 1947.

[19] H. Umezawa, Advanced Field Theory: Micro, Macro, and Thermal Physics, AIP, New York, NY, USA, 1993.

[20] J. Wess and B. Zumino, "A lagrangian model invariant under supergauge transformations," Physics Letters B, vol. 49, no. 1, pp. 52-54, 1974.

[21] A. Capolupo, M. Di Mauro, and A. Iorio, "Mixing-induced spontaneous supersymmetry breaking," Physics Letters, Section A: General, Atomic and Solid State Physics, vol. 375, no. 39, pp. 3415-3418, 2011.

[22] N. E. Mavromatos, S. Sarkar, and W. Tarantino, "D-foaminduced flavor condensates and breaking of supersymmetry in free Wess-Zumino fluids," Physical Review D, vol. 84, Article ID 044050, 2011.

[23] A. Capolupo and G. Vitiello, "Spontaneous supersymmetry breaking probed by geometric invariants," Advances in High Energy Physics, vol. 2013, Article ID 850395, 5 pages, 2013.

[24] J. Anandan and Y. Aharonov, "Geometry of quantum evolution," Physical Review Letters, vol. 65, no. 14, pp. 1697-1700, 1990.

[25] A. Capolupo and G. Vitiello, "Probing Hawking and Unruh effects and quantum field theory in curved space by geometric invariants," Physical Review D, vol. 88, Article ID 024027, 2013.

[26] A. Capolupo, "Probing CPT violation in meson mixing by a noncyclic phase," Physical Review D, vol. 84, no. 11, Article ID 116002, 10 pages, 2011.

[27] M. Blasone, A. Capolupo, E. Celeghini, and G. Vitiello, "Noncyclic phases for neutrino oscillations in quantum field theory," Physics Letters, Section B: Nuclear, Elementary Particle and HighEnergy Physics, vol. 674, no. 1, pp. 73-79, 2009.

[28] S. Midorikawa, "Behavior of supersymmetry at finite temperature," Progress of Theoretical Physics, vol. 73, no. 5, pp. 1245-1257, 1985.

[29] V. V. Lebedev and A. V. Smilga, "Supersymmetric sound," Nuclear Physics, Section B, vol. 318, no. 3, pp. 669-704, 1989.

[30] K. Kratzert, "Finite-temperature supersymmetry: the WessZumino model," Annals of Physics, vol. 308, no. 1, pp. 285-310, 2003. 

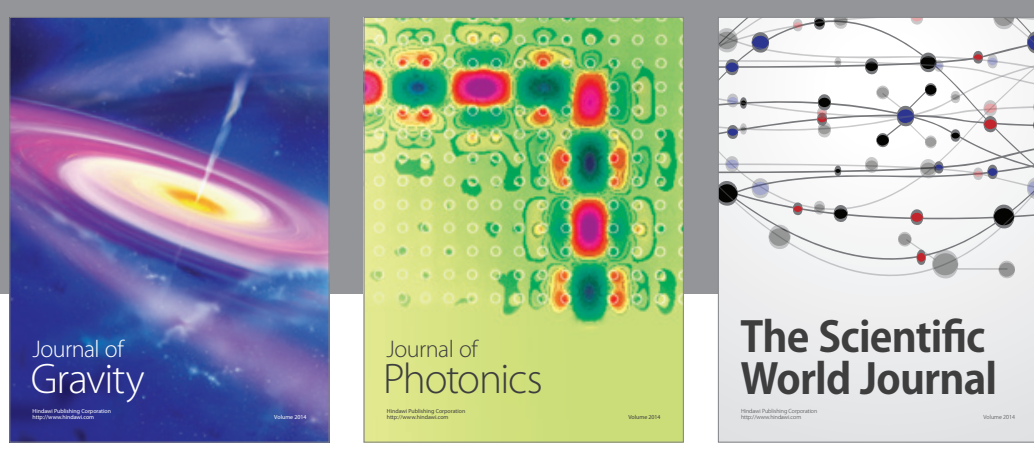

The Scientific World Journal
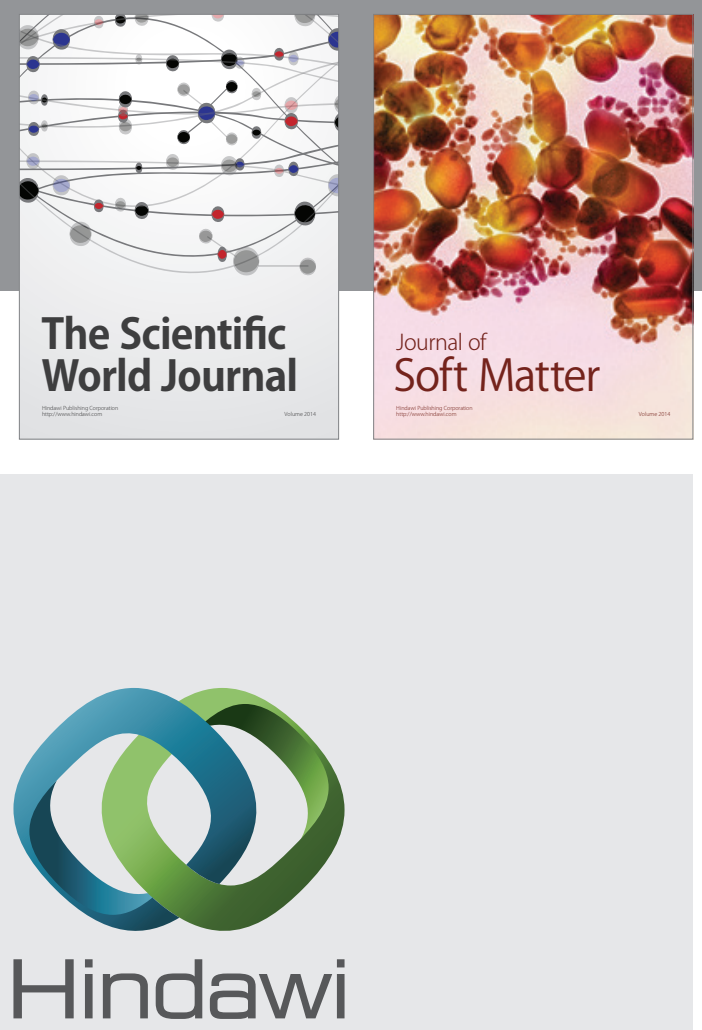

Submit your manuscripts at

http://www.hindawi.com

nternational Journal of

Statistical Mechanics
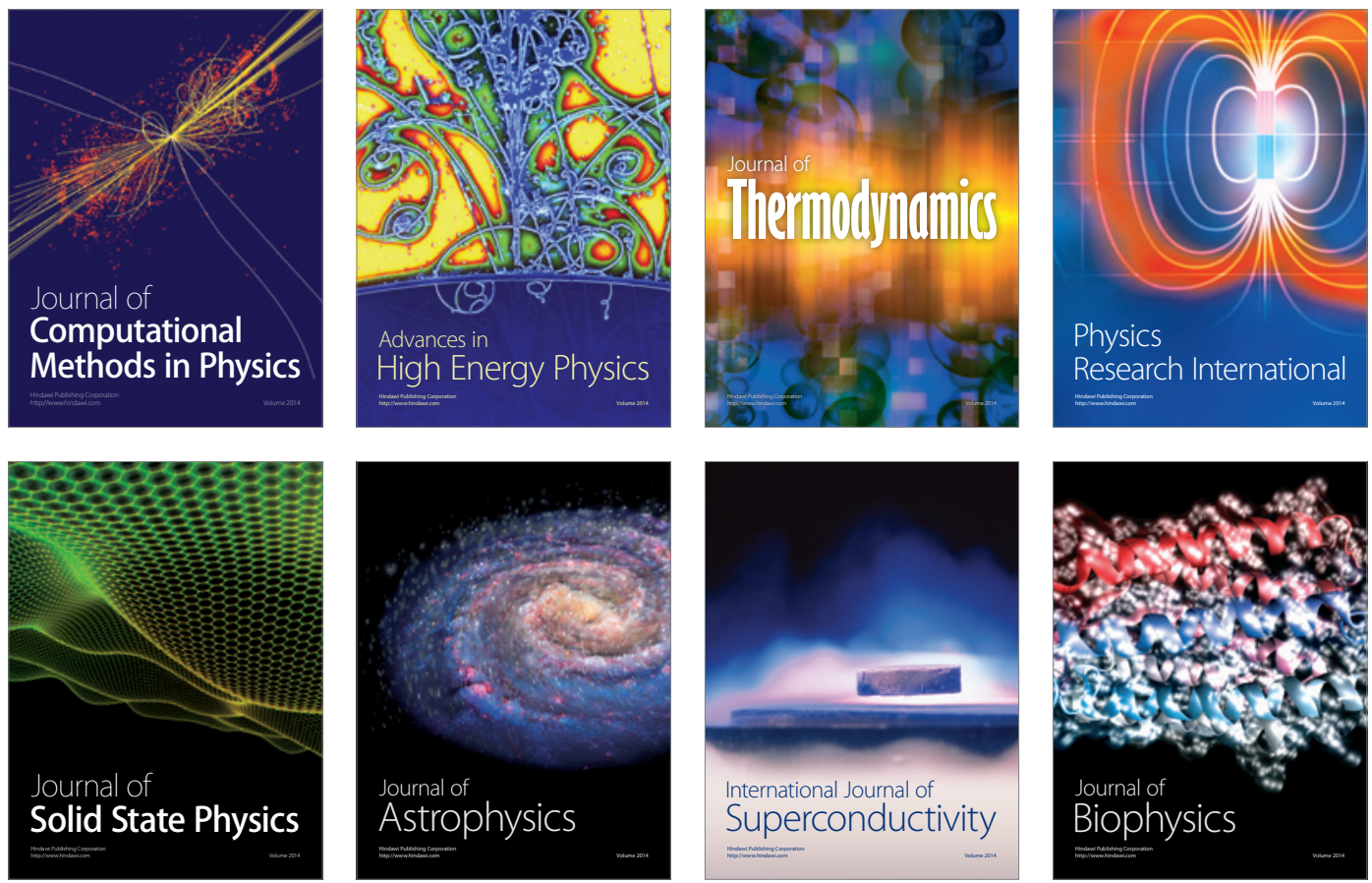
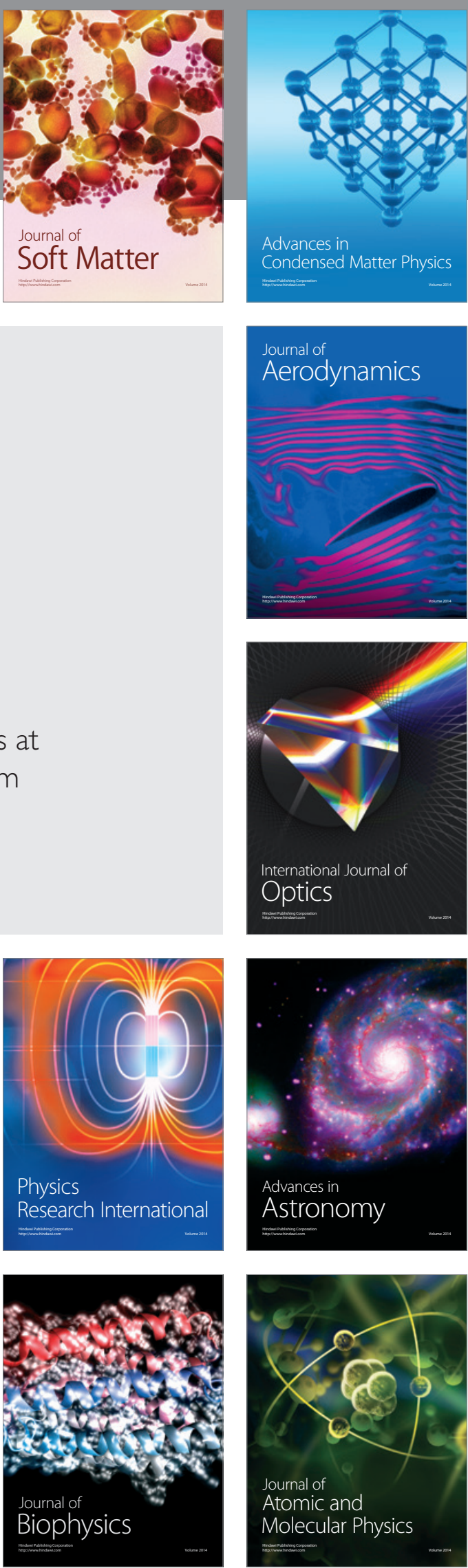